\title{
Modification of flavin adenine dinucleotide in alcohol oxidase of the yeast Hansenula polymorpha
}

\author{
LEONID V. BystryKh, ${ }^{*}$ LUbBert DiJKhuizen and Wim HARDER \\ Department of Microbiology, University of Groningen, Kerklaan 30, 9751 NN Haren, The Netherlands
}

(Received 20 May 1991; revised 18 July 1991; accepted 23 July 1991)

\begin{abstract}
Alcohol oxidase, a major peroxisomal protein of methanol-utilizing yeasts, may possess two different forms of flavin adenine dinucleotide, classical FAD and so-called modified FAD (mFAD). Conversion of FAD into mFAD was observed both in purified preparations of the enzyme and in cells grown in batch and continuous culture. The relative amount of $\mathrm{mFAD}$ in the enzyme varied from 5 to $95 \%$, depending on the growth or storage conditions. The presence of $\mathrm{mFAD}$ led to a slight decrease in $V_{\max }$ and a significant (about one order) decrease in the $K_{\mathrm{m}}$ of alcohol oxidase with respect to methanol. The kinetics of modification measured in purified preparations of the enzyme obeyed first-order kinetics $\left(k=0.78 \mathrm{~h}^{-1}\right)$. The modification process was strongly inhibited by methanol, formaldehyde or hydroxylamine. Modification observed in continuous culture under steady state conditions depended on the dilution rate and could also be described as a spontaneous first-order reaction $\left(k_{\mathrm{app}}=0 \cdot 27 \mathrm{~h}^{-1}\right)$. FAD modification could only be detected in alcohol oxidase and not in other yeast peroxisomal flavoenzymes, such as D-amino acid oxidase from Candida boidinii.
\end{abstract}

\section{Introduction}

Alcohol oxidase is the first enzyme of methanol oxidation in methylotrophic yeasts (for a review see Woodward, 1990). It is an FAD-containing flavoprotein which is localized in peroxisomes. In yeasts grown in methanol-limited chemostats the level of this enzyme may be as high as $60 \%$ of total soluble protein (Roggenkamp et al., 1984). Its synthesis requires coordinate induction of enzymes leading to the production of FAD (Brooke et al., 1986). During growth on methanol the main intracellular role of FAD is therefore that of prosthetic group of alcohol oxidase.

FAD is mainly present in a modified form (mFAD) in alcohol oxidases of various methanol-utilizing yeasts (Sherry \& Abeles, 1985; Bystrykh et al., 1989a). NMR and HPLC analyses have revealed that structural differences between this MFAD and FAD reside in the riboflavin part of the molecule, namely in the $2^{\prime}-3^{\prime}$ carbon atoms of the ribityl side chain of riboflavin (Bystrykh et al., 1991). Previously, we reported on the influence of growth rate on mFAD appearance in alcohol oxidase (Bystrykh et al., 1989a,b). However, nothing is known about the process of mFAD synthesis in yeast cells.

\footnotetext{
Abbreviations: FAD, flavin adenine dinucleotide; mFAD, modified FAD.
}

In this paper we show that modification of FAD occurs when it functions as a prosthetic group of alcohol oxidase, both in whole cells of yeasts and in homogeneous preparations of the enzyme. The results imply that mFAD is derived from FAD and excludes the possibility of separate biosynthetic pathways for these compounds. The presence of mFAD has a pronounced effect on the catalytic properties of alcohol oxidase.

\section{Methods}

Cultivation. Hansenula polymorpha CBS 4732, Hansenula polymorpha. DL-1 and Candida boidinii ATCC 32195 were grown in batch and continuous cultures with $1 \%(\mathrm{v} / \mathrm{v})$ methanol or $0.5 \%$ glucose as described by van der Klei et al. (1990). Cells of Candida boidinii ATCC 32195 were also grown in a chemostat with $0.5 \%(w / v)$ DL-alanine as a carbon source at $D=0.04 \mathrm{~h}^{-1}$.

Enzyme assays. Enzyme activities were determined according to the procedures described by van der Klei et al. (1990). Activity of D-amino acid oxidase was determined in extracts using the same assay mixture as for alcohol oxidase except that D-alanine $(50 \mathrm{~mm})$ was used instead of methanol. Kinetic constants were calculated from initial plots of $v(s)$. using linear regression analysis. One unit of enzyme activity is defined as the amount of enzyme catalysing the conversion of $1 \mu \mathrm{mol}$ of the substrate $\min ^{-1}$.

Enzyme purification. For the analysis of FAD in alcohol oxidase or in D-amino acid oxidase, cells (about $5 \mathrm{~g}$ ) grown on methanol, glucose or DL-alanine were collected by centrifugation and disintegrated in a 
French pressure cell as described by van der Klei et al. (1990) in $10 \mathrm{ml}$

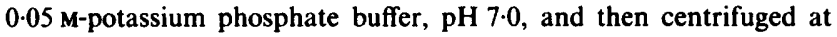
$18000 \mathrm{~g}$. The supernatant was applied to a Superose-6 column $(10 \mathrm{~mm} \times 30 \mathrm{~cm}$, FPLC, Pharmacia) equilibrated with the same buffer. Chromatography was done at a flow rate of $0.5 \mathrm{ml} \mathrm{min}^{-1} ; 1 \mathrm{ml}$ fractions were collected and analysed for enzyme activities and FAD distribution. Alcohol oxidase purified from cells grown on methanol in batch culture and harvested in the exponential growth phase (van der Klei et al., 1990) was used in kinetic experiments for analysis of factors influencing FAD modification. The following effectors were used in these experiments: methanol, $20 \mathrm{mM}$; formaldehyde, $6 \mathrm{mM}$; hydroxylamine, $10 \mathrm{mM}$. The amount of alcohol oxidase in the assay mixtures was $0.78 \mathrm{mg}$ protein, containing $2.6 \mu \mathrm{M}$ total FAD. The oxidase activity was 20 and 2 units, measured with methanol and formaldehyde respectively. Catalase ( 40 units) was added to prevent accumulation of hydrogen peroxide in the reaction mixtures.

FAD analysis. Extraction of FAD was done as described previously (Brooke et al., 1986) by treating samples with $5 \%(w / v)$ trichloroacetic acid followed by centrifugation in a Sorvall $5 B$ centrifuge at $18000 \mathrm{~g}$ for $10 \mathrm{~min}$ at $4{ }^{\circ} \mathrm{C}$ (TCA extracts). The supernatants were used for the determination of the FAD contents by analytical HPLC, using a $\mu$ Bondapack C18 $(3.9 \times 300 \mathrm{~mm}$, Waters $)$ column and a buffer solution (buffer A) containing $0.1 \mathrm{M}$-sodium formate/formic acid, $\mathrm{pH} 2 \cdot 7$, acetonitrile $\left(100 \mathrm{ml} \mathrm{l}^{-1}\right)$ and methanol $\left(20 \mathrm{ml} \mathrm{l}^{-1}\right)$. In some experiments a buffer solution (buffer B) as described by Bystrykh et al. (1989a) was used. Buffer $\mathrm{B}$ (final $\mathrm{pH} 2 \cdot 5-2 \cdot 7$ ) was prepared by adding $2 \mathrm{~g} \mathrm{H}_{3} \mathrm{PO}_{4}$ to $0.356 \mathrm{M}-\mathrm{K}_{2} \mathrm{HPO}_{4}$, with acetonitrile $\left(90 \mathrm{ml} \mathrm{l}^{-1}\right)$ and methanol $\left(50 \mathrm{mll}^{-1}\right)$. The flow rate was $0.5 \mathrm{ml} \mathrm{min}{ }^{-1}$ (buffer A) or $0.7 \mathrm{ml} \mathrm{min}^{-1}$ (buffer B); detection was at $280 \mathrm{~nm}$ using a $0.05 \mathrm{~A}$ full-scale setting on the detector. The amount of FAD and MFAD was calculated via integration of peak areas at $280 \mathrm{~nm}$. A commercial solution of FAD (Sigma) was used as a standard.

\section{Results and Discussion}

\section{Comparison of $m F A D$ from different sources}

HPLC analysis of TCA extracts revealed that modification of FAD occurs in different methylotrophic yeasts, namely Hansenula polymorpha CBS $4732, H$. polymorpha DL-1 and Candida boidinii ATCC 32195, resulting in the same final product. Commercial preparations of alcohol oxidase from Pichia pastoris (Provesta Corp., USA) also contained mFAD. The presence of mFAD could only be detected in alcohol oxidase and not in any other (peroxisomal) flavoenzyme of methylotrophic yeasts, e.g. D-amino acid oxidase from C. boidinii (Fig. 1).

The mFAD content of methanol-grown cells of $\boldsymbol{H}$. polymorpha varied depending on the growth conditions, e.g. batch or continuous cultures. A kinetic analysis of the alcohol oxidase purified from these cells showed that mFAD confers upon the enzyme a higher affinity for methanol and a lower maximum acitivity. The enzyme preparation obtained from batch culture ( $1 \%$ methanol) cells contained $15 \%$ of mFAD and displayed a $V_{\max }$ of $10.9 \mathrm{U} \mathrm{mg}^{-1}$ and a $K_{\mathrm{m}}$ of $2.1 \mathrm{~mm}$. The alcohol oxidase purified from methanol-limited chemostat $\left(D=0 \cdot 1 \mathrm{~h}^{-1}\right)$ cells contained $80 \%$ of mFAD, exhibiting $V_{\max }$ and $K_{\mathrm{m}}$ values of $5.5 \mathrm{U} \mathrm{mg}^{-1}$ and $0.16 \mathrm{mM}$, respectively.
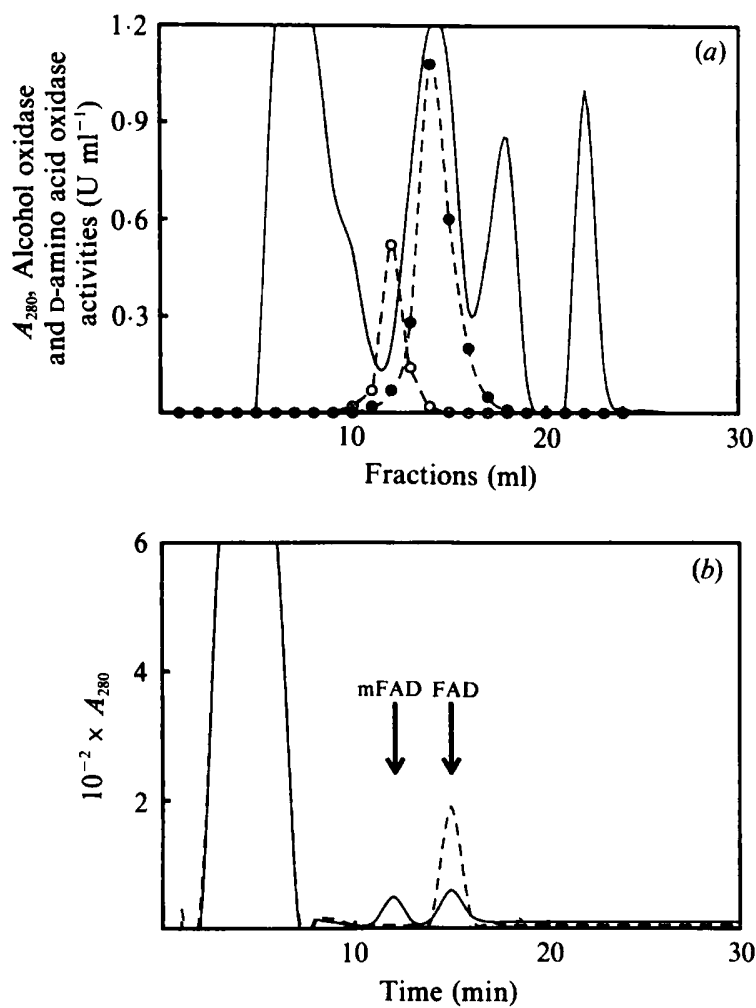

Fig. 1. Presence of mFAD in alcohol oxidase and D-amino acid oxidase from cells of $C$. boidinii ATCC 32195 grown in continuous culture on DL-alanine. (a) Separation of the enzymes by FPLC on Superose-6; -,$A_{280} ;---$, activities of alcohol oxidase $(O)$ and Damino acid oxidase (O). (b) HPLC analysis of FAD extracted from alcohol oxidase (-) or from D-amino acid oxidase (---). Equal FPLC fraction volumes of the enzymes were applied for HPLC analysis (using buffer B).

FAD modification, however, also occurred during storage of alcohol oxidase preparations (see below), resulting in similar changes in their kinetic characteristics. The FAD content of alcohol oxidase in cell samples and purified enzyme preparations was determined immediately after harvesting and/or purification in order to minimize interference of this continuing modification with our studies.

\section{Synthesis of mFAD in batch culture}

Negligible amounts of mFAD were found both in whole cells and in purified preparations of alcohol oxidase during exponential growth of $H$. polymorpha CBS 4732 (and other methylotrophic yeasts, not shown) in batch cultures at high methanol concentrations. Appearance of mFAD was observed after complete consumption of methanol from the cultivation medium, which almost stoichiometrically correlated with a decrease in unmodified FAD levels in whole cells (Fig. 2). 

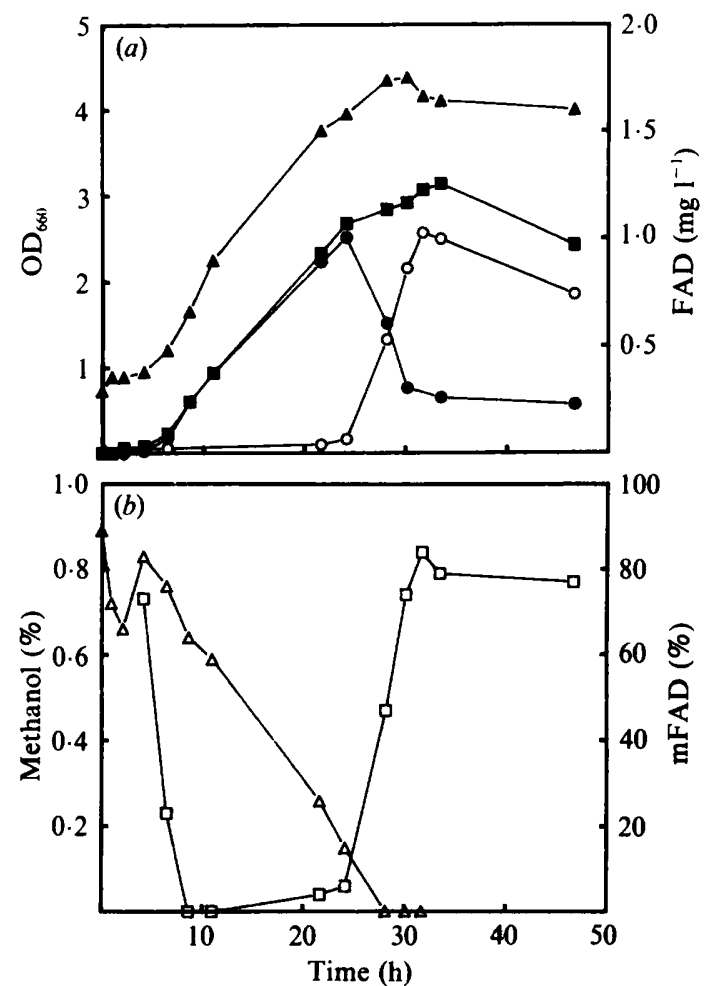

Fig. 2. Modification of FAD during growth of $\boldsymbol{H}$. polymorpha CBS 4732 in batch culture with $1 \%$ methanol. (a) $\triangle, \mathrm{OD}_{660} ; \square$, total FAD; 0 , unmodified FAD; $O$, mFAD. (b) $\triangle$, Methanol; $\square$, percentage mFAD. Cells were pre-grown under the same conditions till reaching the stationary phase (compare percentage mFAD at 5 and $45 \mathrm{~h}$ ).

\section{Modification of FAD in homogeneous preparations of alcohol oxidase}

During purification of alcohol oxidase from cells of $H$. polymorpha CBS 4732 grown in batch culture the $\mathrm{mFAD} / \mathrm{FAD}$ ratio in the enzyme samples increased. Storage of this alcohol oxidase preparation (containing $60 \%$ ammonium sulphate) at $4{ }^{\circ} \mathrm{C}$ or at $30^{\circ} \mathrm{C}$ clearly resulted in further modification of the enzyme-bound FAD. The modification reaction was not observed with free FAD under the same incubation conditions. This implies that the appearance of mFAD is based on conversion of FAD into mFAD when it is present as a prosthetic group of alcohol oxidase. The above observations exclude any separate de novo synthesis of these two forms of FAD in methylotrophic yeasts.

As shown in Fig. 3, addition of either formaldehyde or methanol to purified alcohol oxidase preparations inhibited the modification reaction. Both methanol and formaldehyde were completely consumed from the assay mixtures within the first few minutes, but the inhibitory effect of the substrates was maintained throughout the whole incubation period $(2 \mathrm{~h})$. Addition of hydroxylamine had the same effect as the enzyme substrates,
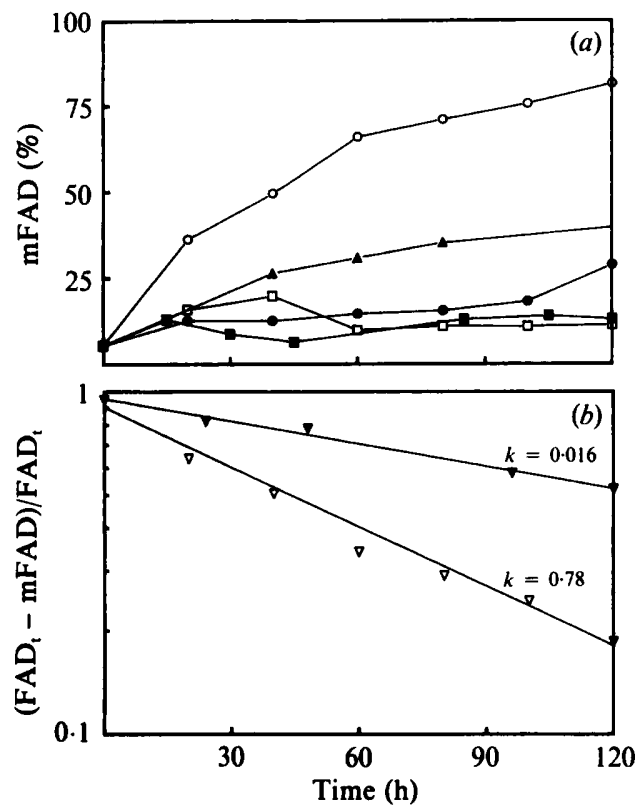

Fig. 3. Primary (a) and logarithmic (b) plots of the modification of FAD in a purified preparation of alcohol oxidase at $37^{\circ} \mathrm{C}$ [all data in $(a), \nabla$ in $(b)]$ and $4^{\circ} \mathrm{C}[\nabla$ in (b) $]$. (a) O, Free enzyme; $O$, with $6 \mathrm{mM}$ formaldehyde; $\square, 20$ mM-methanol; $\square, 10$ mM-hydroxylamine; $\triangle$, under nitrogen flow (anaerobic conditions). Modification constants $(k)$ for the free enzyme are given in part $(b)$. Alcohol oxidase was purified from cells of $H$. polymorpha CBS 4732 grown on methanol in batch culture.

although its oxidation did not result in accumulation of formaldehyde or formate in the assay mixture. The presence of hydroxylamine leads to formation of the semiquinone form of FAD in the enzyme (Geissler \& Hemmerich, 1981; Geissler et al., 1986), whereas addition of true substrates leads to the fully reduced form of FAD. Incubation of alcohol oxidase preparations under a nitrogen flow (anaerobic conditions) also inhibited the modification reaction (Fig. $3 a$ ).

The logarithmic plot of the modification reaction versus time is linear (Fig. $3 b$ ). The modification constant for the free enzyme was $0.78 \mathrm{~h}^{-1}$ and $0.016 \mathrm{~h}^{-1}$ at $37^{\circ} \mathrm{C}$ and $4{ }^{\circ} \mathrm{C}$, respectively. Reduction of the enzyme by twoelectron (methanol, formaldehyde) or one-electron (hydroxylamine) donors thus inhibits the FAD modification reaction. Since removal of oxygen from the assay mixture caused the same effect, it appears likely that the oxidized form of FAD is the substrate in the modification reaction. It has been suggested that the semiquinone form is stable upon its oxidation by oxygen in alcohol oxidase (Mincey et al., 1980; Geissler \& Hemmerich, 1981; Geissler et al., 1986; Sherry \& Abeles, 1985; Hopkins \& Muller, 1987). According to our knowledge the enzyme may also exist in a stable reduced form (L. V. Bystrykh, unpublished). The presence of these various 
stable forms and their slow conversion to the oxidized form may explain the prolonged inhibitory effects of the compounds tested.

\section{Steady-state kinetics of the modification reaction in whole} cells

Analysis of the kinetics of FAD modification in purified alcohol oxidase preparations described above revealed that the modification reaction occurs spontaneously and obeys first-order kinetics in the absence of substrates. The inhibitory effects caused by substrates remained even after their complete exhaustion from the reaction mixture and therefore cannot be described as a function of substrate concentration.

To compare the reaction of FAD modification in vivo and in vitro, the following theoretical approach was introduced. When the change of total FAD is negligible (for instance, in purified enzyme preparations or in the stationary growth phase) the transformation of FAD into mFAD is described by first-order kinetics.

In chemostat-grown cells FAD modification can be described through the following rates $(V)$ of production of different forms of FAD and their concentrations: total, $V_{\mathrm{ft}}$, [FAD $]$; unmodified, $V_{\mathrm{f}}$, [FAD]; modified, $V_{\mathrm{fm}}$ [mFAD]. The equations given below include real $(V)$ and observed $\left(V^{\prime}\right)$ rates of FAD productions, related via the dilution rate $(D)$ and the FAD modification constant $(k)$.

$$
\begin{aligned}
& V_{\mathrm{ft}}^{\prime}=V_{\mathrm{ft}}-D\left[\mathrm{FAD}_{\mathrm{t}}\right] \\
& V_{\mathrm{f}}^{\prime}=V_{\mathrm{ft}}-(k+D)[\mathrm{FAD}] \\
& V_{\mathrm{fm}}^{\prime}=V_{\mathrm{ft}}^{\prime}-V_{\mathrm{f}^{\prime}}^{\prime}
\end{aligned}
$$

Two transformations of these equations are applied in the experiments. These are required to estimate the value of $k$, the modification constant, and to analyse the fitness of the modification reaction to the model proposed above.

$$
\begin{aligned}
k= & V_{\mathrm{fm}} /[\mathrm{FAD}]+D[\mathrm{mFAD}] /[\mathrm{FAD}] \\
& {\left[\mathrm{FAD}_{\mathrm{t}}\right] /[\mathrm{mFAD}]=1+D / k }
\end{aligned}
$$

Equation (2) can be used for both steady state and transient state chemostat cultures, whereas equation (3) can be applied to steady state conditions only.

An analysis of a function of the amount of mFAD versus dilution rate with the two strains of $H$. polymorpha shows a reasonably good correlation between measured (data points) and predicted (lines) relative concentrations of mFAD versus $D$ [equation (3), Fig. 4], except for strain DL-1 at the highest $D$ value which does not result in a stable steady state. This supports the relevance of the proposed model for steady state conditions.

For the two $H$. polymorpha strains, the specific rates of modification by whole cells and purified alcohol oxidase
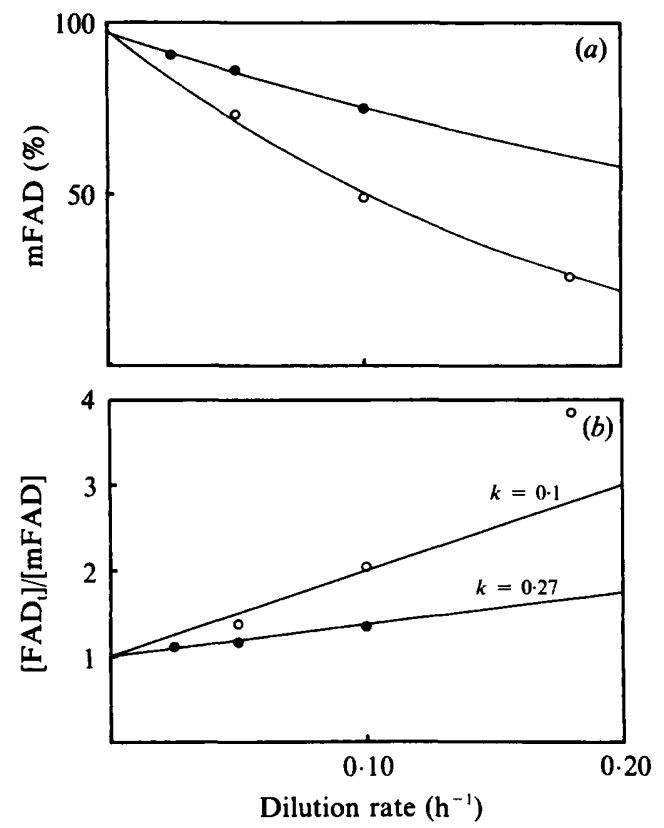

Fig. 4. Primary (a) and reciprocal (b) plots of relative mFAD concentrations versus dilution rate in cells of $\boldsymbol{H}$. polymorpha DL-1 (O) and CBS 4732 (O) grown in continuous cultures on methanol. Modification constants measured at steady state conditions are indicated. Lines were drawn (b) according to equation (3).

preparations are different. The modification constants for strains DL-1 and CBS 4732 were $0.1 \mathrm{~h}^{-1}$ and $0.27 \mathrm{~h}^{-1}$ respectively, which is significantly less than the corresponding modification constant measured with purified alcohol oxidase preparations $\left(0.78 \mathrm{~h}^{-1}\right)$. This suggests that the modification process is influenced by, for instance, the low concentration of methanol in the chemostat culture, or significantly different rates of alcohol oxidase synthesis and degradation (turnover effect) with respect to dilution rate.

\section{Modification of FAD under non-steady-state conditions}

Transfer of cells of $H$. polymorpha CBS 4732 from batch (late-exponential growth phase) to chemostat culture growth conditions $\left(D=0.025 \mathrm{~h}^{-1} ; \mathrm{Fig} .5\right)$ resulted in a large increase in mFAD levels, but not of unmodified FAD. During the transition period the $k$ value fluctuated between 0.06 and $0.67 \mathrm{~h}^{-1}$ before approaching the constant level $\left(0.27 \mathrm{~h}^{-1}\right)$ already observed for methanollimited steady state conditions (Fig. 4). Transfer of cells from glucose- to methanol-limited growth conditions $\left(D=0.05 \mathrm{~h}^{-1}\right)$ resulted in a similar increase in mFAD levels and fluctuations in the $k$ value (Fig. 6).

The maximal growth rate observed with $H$. polymorpha CBS 4732 in methanol batch cultures was $0.128 \mathrm{~h}^{-1}$, resulting in very low mFAD levels (Fig. 2). 

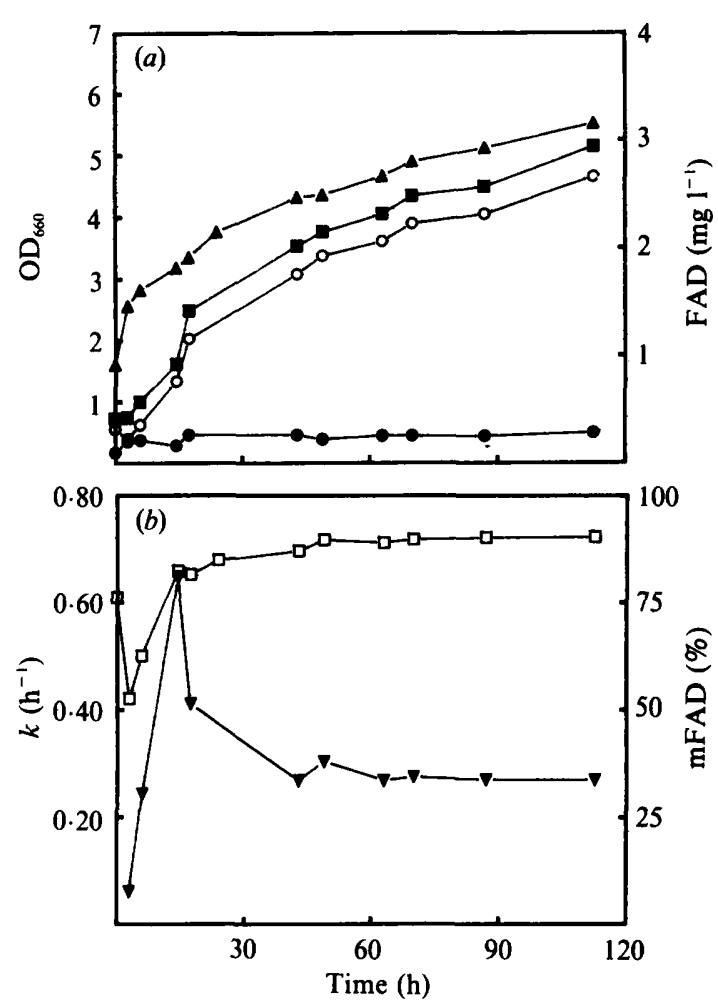

Fig. 5
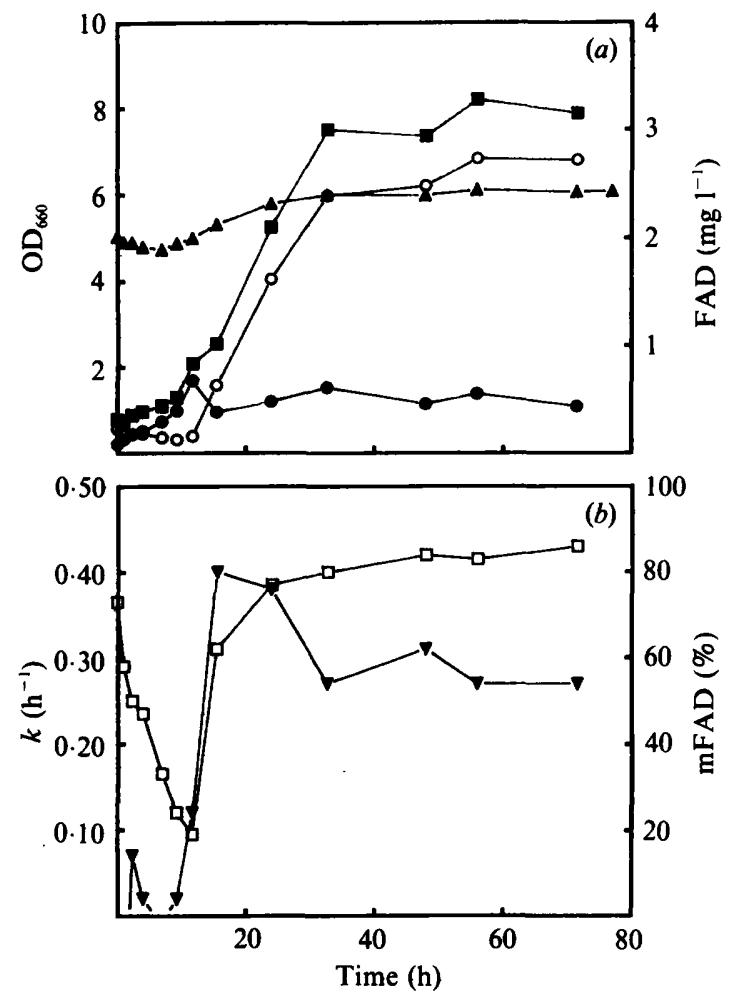

Fig. 6

Fig. 5. Modification of FAD in cells of $H$. polymorpha CBS 4732 during transfer from batch growth on $1 \%$ methanol to conditions of methanol limitation in continuous cultures $\left(1 \%\right.$ methanol at $\left.D=0.025 \mathrm{~h}^{-1}\right) .(a) \Delta, \mathrm{OD}_{660} ; \mathbf{Q}$, total FAD;, unmodified FAD; O, mFAD. (b) $\square$, percentage mFAD; $\nabla$, modification constant $(k)$.

Fig. 6. Modification of FAD in carbon-limited continuous culture cells of $H$. polymorpha CBS 4732 during transition from $0.5 \%$ glucose to $1 \%$ methanol at $D=0.05 \mathrm{~h}^{-1}$. (a) $\triangle, \mathrm{OD}_{660} ; \square$, total FAD; 0 , unmodified FAD; O, mFAD. (b) $\square$, percentage mFAD; $\nabla$, modification constant $(k)$.

Under steady state chemostat conditions this growth rate would yield an mFAD level of $68 \%$ (Fig. 4). The data show that the rate of FAD modification is low in batch cultures and transient state continuous cultures. During the transition experiments a slight accumulation of methanol (up to $0 \cdot 2 \%$ ) was observed in the first $2-4 \mathrm{~h}$. It is therefore concluded that the (initially) low rates of FAD modification were caused by the presence of methanol, but not by differences in growth rates.

The results presented thus indicate that the kinetic properties of alcohol oxidase are dependent on the presence of different forms of FAD. The mFADcontaining enzyme exhibits significantly higher affinity towards methanol, retaining most of its activity. Low substrate concentrations are favourable for FAD modification to occur. The modification of FAD thus is of physiological importance and it may represent an unusual mechanism for adaptation to substrate-limited growth conditions.

Modification of FAD in alcohol oxidase and the variability of the kinetic properties of this enzyme is a widespread and uniform phenomenon in methylotrophic yeasts (Bystrykh et al., 1989a, $b$; Sherry \& Abeles, 1985). The FAD modification rate in $H$. polymorpha CBS 4732 cells may vary (depending on the growth conditions) from 0.01 to $0.67 \mathrm{~h}^{-1}$, which is always below the modification rate observed in vitro with purified alcohol oxidase. Under conditions of steady state growth in chemostats the modification rate is $0.27 \mathrm{~h}^{-1}$ (Fig. 4). It is unlikely that the observed differences between modification constants, about $0.5 \mathrm{~h}^{-1}$, could be assigned to synthesis and degradation of alcohol oxidase. Rates of enzyme degradation varied between 0.02 and $0.12 \mathrm{~h}^{-1}$ in late-stationary phase cells after batch growth on methanol, and in transitions from methanol batch to glucoselimited chemostat culture $\left(D=0.075 \mathrm{~h}^{-1}\right)$ conditions, respectively. These values are considerably lower than the differences in $k$ values observed. The observations, however, can again be explained by the inhibitory effect of methanol on flavin modification. When using the $K_{\mathrm{m}}$ value for alcohol oxidase with unmodified FAD for estimation of substrate-free enzyme, calculations show 
that an intracellular concentration of methanol of about $0.01 \%$ is sufficient to decrease the observed modification constant in vivo from 0.78 to $0.27 \mathrm{~h}^{-1}$.

The observed value of the modification constant is higher then the maximal growth rate of yeasts on methanol, which is usually below $0 \cdot 18-0 \cdot 2 \mathrm{~h}^{-1}$. Stable growth of yeasts on methanol is maintained at lower $D$ values, around $0 \cdot 1 \mathrm{~h}^{-1}$ (Egli et al., 1983, 1986). As a result, modification of FAD in growing cells follows the 'all-or-nothing' principle, yielding either mostly normal (in the presence of methanol, no correlation with growth rate) or modified FAD (at negligible methanol concentrations, depending on dilution rate).

\section{References}

Brooke, A. G., DiJkHUizen, L. \& HARDer, W. (1986). Regulation of flavin biosynthesis in the methylotrophic yeast Hansenula polymorpha. Archives of Microbiology 145, 62-70.

Bystrykh, L. V., Romanov, V. P., Steczko, J. \& Trotsenko, Y. A. $(1989 a)$. Catalytic variability of alcohol oxidase from the methylotrophic yeast Hansenula polymorpha. Biotechnology and Applied Biochemistry 11, 184-192.

ByStRYKH, L. V., Dvorakova, J. \& Volfova, O. (1989b). Alcohol oxidase of methylotrophic thermo- and acidotolerant yeast Hansenula sp. Folio Microbiologica 34, 233-237.

Bystryki, L. V., KellogG, R. M., KRUizinga, W., Dijkhuizen, L., Harder, W., Vervoort, J. \& van Berkel, W. J. H. (1991). Structural and kinetic analysis of flavin adenine dinucleotide modification in alcohol oxidase from methylotrophic yeasts. In Flavins and Flavoproteins 1990. Proceedings of the 10th International Symposium, pp. 197-200. Edited by B. Curti, S. Ronchi \& G. Zanetti. Berlin: Walter de Gruyter.
Egli, Th., Lindley, N. \& QUayle, J. R. (1983). Regulation of enzyme synthesis and variation of residual concentration during carbonlimited growth of Kloeckera sp. 2201 on mixtures of methanol and glucose. Journal of General Microbiology 129, 1269-1281.

EGLI, Th., Bosshard, C. \& HAMER, G. (1986). Simultaneous utilization of methanol-glucose mixtures by Hansenula polymorpha in a chemostat: influence of dilution rate and mixture composition on utilization pattern. Biotechnology and Bioengineering 28, 1735-1741.

Geissler, J. \& Hemmerich, P. (1981). Yeast methanol oxidases: an unusual type of flavoprotein. FEBS Letters 126, 152-156.

Geissler, J., Ghisla, S. \& Kroneck, P. H. (1986). Flavin-dependent alcohol oxidase from yeast. Studies on the catalytic mechanism and inactivation during turnover. European Journal of Biochemistry 160, 93-100.

HopKINS, T. \& MULLER, F. (1987). Biochemistry of alcohol oxidase. In Proceedings of the 5th International Symposium on Microbial Growth on Cl compounds, pp. 150-157. Edited by H. W. van Verseveld \& J. A. Duine. Dordrecht: Martinus Nijhoff.

VAN DER K LEI, I. J., BYSTRYKh, L. V. \& HARDER, W. (1990). Alcohol oxidase from Hansenula polymorpha CBS 4732. Methods in Enzymo$\log y 188,420-427$.

Mincey, T., Taylern, G., Mildvan, A. S. \& Abeles, R. H. (1980). Presence of a flavin semiquinone in methanol oxidase. Biochemistry 77, 7099-7101.

Roggenkamp, R., Janowicz, Z., Stanikowski, B. \& Hollenberg, C. (1984). Biosynthesis and regulation of the peroxisomal methanol oxidase from the methylotrophic yeast Hansenula polymorpha. Molecular and General Genetics 194, 489-493.

SHERRY, B. \& ABELES, R. H. (1985). Mechanism of action of methanol oxidase, reconstitution of methanol oxidase with 5-deazaflavin, and inactivation of methanol oxidase by cyclopropanol. Biochemistry 24, 2594-2605.

WOODWARD, J. R. (1990). Biochemistry and applications of alcohol oxidase from methylotrophic yeasts. In Advances in Autotrophic Microbiology and One-Carbon Metabolism, vol. 1, pp. 205-238. Edited by G. A. Codd, L. Dijkhuizen \& F. R. Tabita. Dordrecht: Martinus Nijhoff. 